\title{
An algorithm for estimating the detection efficiency of a
}

\section{lightning location system}

\author{
Haibo $\mathrm{Hu}^{1}$, Xiya Zhang ${ }^{1}$
}

\section{Institute of Urban Meteorology, CMA, Beijing 100089, China}

Abstract This paper puts forward an algorithm for estimating the detection efficiency (DE) of a lightning location system (LLS). The algorithm can be applied to lightning flash/stroke density correction (e.g., cloud-to-ground (CG) lightning flash/stroke density) and LLS performance evaluation. A lightning strike density correction for DE promotes the applicability of the LLS data. Fundamentally, the generalized extreme value (GEV) distribution was found to best fit the probability distribution of the signal strengths of the lightning observed by the ADTD detectors in Beijing, China. With respect to this probability distribution, we estimated the single-station acceptance damped by the uneven underlying land surface conductivity. Accounting for the multi-detector location modes supported by single-station acceptance, the iterative algorithm was applied for deducing the DE of a LLS. In this case study, the DE estimates of the ADTD network were lower in the mountainous areas than in the plains. These lower estimates can be due to the low underlying conductivity of the mountainous areas, which creates a high attenuation effect on the lightning electromagnetic signals, and the greater distances from the lightning detectors. Subsequently, the cloud-to-ground (CG) lightning flash/stroke density derived from the ADTD data was corrected for the DEs. The results indicated that the CG lightning flash/stroke densities in the northern and northwestern mountainous areas are lower than that in the highly urbanized plains. This anomaly is due to the effects of the increased roughness of the underlying land surfaces, enhanced aerosols, urban heat island (UHI), and intensifying thunderstorm activities in urban areas, but this anomaly is not likely related to the DE discrepancy.

Key words Lighting location system, Detection efficiency, Cloud-to-ground lightning 
1 flash/stroke density.

\section{Introduction}

Lightning location systems (LLSs) capture natural lightning electromagnetic signals and consequently locate lightning sources. LLS observations can be used to derive and retrieve lightning parameters that indicate lighting characteristics, e.g., the cloud-to-ground (CG) lightning flash density, the positive/negative CG flash/stroke density, the percent of positive/negative flashes, the first stroke negative and positive peak currents, and the multiplicity for negative and positive flashes (Orville et al. 2002; Orville et al. 2001; Rudlosky and Fuelberg 2010; Taszarek and Czernecki 2015). These types of data have been widely used in weather forecasting, severe weather warning systems and climatological analysis, e.g., assimilation in weather forecasting models (e.g., Fierro et al. 2011; Fierro and Reisner 2011), synoptic analysis of thunderstorms (e.g., Changnon 1993; Cummins and Murphy 2009), presentations of lightning characteristics (e.g., Schulz et al. 2005; Drüe et al. 2007; Mäkelä et al. 2010), lightning climatology and climate pattern recognition (e.g., Antonescu and Burcea 2010; Williams et al. 2005; Taszarek et al. 2015; Etherington and Perry 2017), and lightning risk assessment (Hu et al. 2014). However, a serious problem emerges when a LLS provides an uneven detection efficiency (DE) within the detectable distance or even a very low DE at the edges of the detectable distance (Naccarato and Pinto 2009), which greatly restricts the applicability of the LLS data (Hu et al. 2014; Yao et al. 2012).

DE is determined by the performance and sensitivity of the sensors, the sensor network geometry, the underlying ground conductivity (Schütte et al. 1988; Naccarato and Pinto 2009; Mäkelä et al. 2010), etc. The unevenly distributed DEs contribute to LLS data uncertainty and ruin data validity (Williams 2005; Yao et al. 2012; Hu et al. 2014). Thus, it is desirable to further develop algorithms to estimate the DE, which can be applied for correcting the lightning flash/stroke density derived from the LLS data.

Moreover, such an algorithm is useful in LLS performance evaluation and network deployment validation and would help to determine optimal detector 

originally intended to test network deployment optimization and network performance and eventually became applicable to improve LLS data quality. Schütte et al. (1987, 1988) suggested that the lightning impulse signal strength (LISS) weakens linearly with propagation distance and confirmed the Weibull probability distribution of LISSs received by a single station within a detectable distance. Naccarato and Pinto (2009) estimated the DE of the Brazilian lightning detection network (BrasilDAT) based on the individual sensor DE probability functions, which were derived from a large amount of CG stroke data provided by the network.

These applicable approaches obviously possess reference values in the aftermath of $\mathrm{DE}$ estimation. Note that the precedent work can be cited as a referential source; however, it is inappropriate to simply copy the methods of earlier researchers if considering different data resources and circumstances. For example, the Weibull distribution of LISSs identified by Schütte et al. (1987, 1988) only reflected the lightning characteristics observed by the European LLS network at that time. However, our study revealed that the probability distribution of the LISSs detected by the ADTD sensors deployed around Beijing, China, would fit the generalized extreme value (GEV) distribution, rather than the Weibull distribution. This finding demonstrates the necessity of modifying the precedent methodology to estimate single-station acceptance.

Moreover, the DE and location accuracy of a LLS can be influenced by the 22 lightning location mode, which is the number of detectors synchronously receiving the lightning electromagnetic signal in one lightning location. The algorithm should consider the multi-detector location mode related to the number of detectors in the lightning location. Usually, at least two magnetic direction finder (MDF) detectors and three time-of-arrival (TOA) detectors are needed in each lightning location (Schütte et al. 1988). As observed by a LLS network with newly upgraded IMPACT detectors (combined MDF and TOA technology), a lightning source can be located with signals synchronously detected by two or more such detectors (Bourscheidt et al. 2012). With multi-detector location modes, here, an iterative algorithm is introduced 
to derive the DE from single-station acceptance.

Underlying surface conductivity plays an important role in damping lightning signals and eventually influences the DE (Honma et al. 1998; Cummins et al. 2005; Rudlosky and Fuelberg 2010). Schütte et al. (1987) suggested that land surface damping influences both the DE and location accuracy and that the TOA network appears more sensitive to land surface damping. At this point, the algorithm should consider the damping effect on signal strength, which is induced by land surface conductivity.

The algorithm was constructed by following the abovementioned fundamentals. In this methodology, the LISSs were calculated using a linear signal propagation model suggested by Cummins et al. (1995). The damping coefficients in a grid system were estimated by addressing the land surface conductivity of specific land use and land cover (LULC) types. After correcting the signal strength magnitudes of the damping factor, we identified the GEV probability distribution of the LISSs. Based on this probability density function (PDF), we estimated the single-station acceptance of lightning source signals and ultimately calculated the grid DEs using an iterative algorithm. We corrected the CG lightning stroke density for the DEs and found an improvement in the estimates of the CG lightning stroke density in comparison with the uncorrected density.

Admittedly, the uncertainty cannot be reduced completely through DE estimation because the DE estimation fundamentals involve simulating signal attenuation propagated over certain distances and dampening due to land surface conductivity. The improvement in the LLS data quality (e.g., DE and location accuracy) basically depends on the implementation of optimal detector deployments and detector upgrading, and DE estimates are critical in promoting the applicability of the LLS data.

\section{Data description}

The LLS data collected from 2007-2016 by the ADTD (Advanced TOA and Direction system, where TOA denotes time-of-arrival) network deployed by the China 
distribution of the LISSs and 2) derive the CG flash/stroke density, which is corrected for the DE. These data include the time, location, amperage and polarity of the CG lightning strokes.

The ADTD consists of more than 301 sensors (as of March 2011) in China (Yao et al. 2012). In Beijing, 9-14 ADTD-1 sensors (improved IMPACT (combined MDF and TOA) sensors) can detect $1-450 \mathrm{kHz}$ (the low-frequency (LF) band) lightning sources (Fig. 1). The ADTD-1 sensors use the combined MDF and time-of-arrival

8 (TOA) method for position retrieval (Ma 2015). In this method, if a lightning source

9 is only detected by two ADTD-1 sensors, the algorithm uses one TOA hyperbolic

10 curve and two MDF vectors to retrieve the position. If a lightning source is detected

11 by three sensors in a non-duplicate region, the TOA algorithm is used to retrieve the

12 position directly. In contrast, if the TOA is the first to find a duplicate location, then the MDF method is used to identify the true location. If a lightning event is detected by four or more sensors, a TOA least squares method is used to find a more precise location. Thus, a lightning location detected by four or more sensors is more precise than that detected by fewer sensors. In the ADTD data, the percentage of lightning sources reported by four or more sensors relative to the total number of detected sources is $63.3 \%$. Meanwhile, the ADTD-observed $+\mathrm{CG}$ and $-\mathrm{CG}$ lightning peak currents are in the ranges of $0.08 \mathrm{kA}$ to $995.9 \mathrm{kA}$ and $0.258 \mathrm{kA}$ to $992.6 \mathrm{kA}$, respectively (Fig. 1).

The ADTD sensor manufacturers claim that the DE of the ADTD sensors could 22 be $90 \%$ at distances between 300 and $600 \mathrm{~km}$, with a median location accuracy error of $1 \mathrm{~km}$. However, only the flash DE can be $90 \%$, and the stroke detection efficiency 24 (SDE) is lower. The first stroke peak current in a multiple-stroke CG flash can be greater than twice its subsequent stroke peak current (Rakov and Uman 1990). Thus, the sensors can capture the first larger peak stroke but miss the weaker subsequent stroke (Rudlosky and Fuelberg 2010). Moreover, some weak CG strokes (including single-stroke CG flashes) cannot be detected due to the signal attenuation induced by long-distance propagation and damping effects in lower conductivity mountainous regions (Schütte et al. 1988). 
Nat. Hazards Earth Syst. Sci. Discuss., https://doi.org/10.5194/nhess-2017-307

Manuscript under review for journal Nat. Hazards Earth Syst. Sci.

Discussion started: 25 September 2017

(C) Author(s) 2017. CC BY 4.0 License.

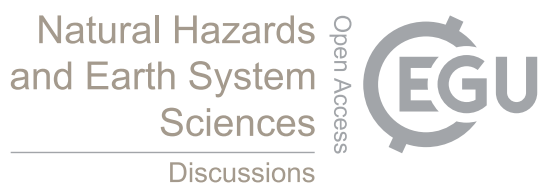

$-6-$

1 We estimated the SDEs of the ADTD in a grid system $(1 \mathrm{~km} \times 1 \mathrm{~km}$, see Fig. 2)

2 and corrected the lightning stroke density using the SDE. The SDE estimates

3 approximate those of the U.S. NLDN (National Lightning Detection Network) in

4 1998, which was reported to be $62 \%$ (Idone et al. 1998). Hence, the DE level of the

5 ADTD is equivalent to that of the NLDN, at least in 1998, suggesting that a

6 considerable potential for improvement remains in terms of network upgrades and that

7 DE estimation is necessary for promoting the applicability of the ADTD data.

(a)

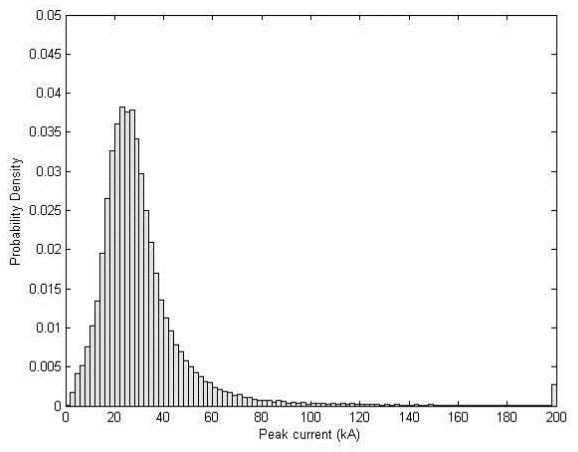

(b)

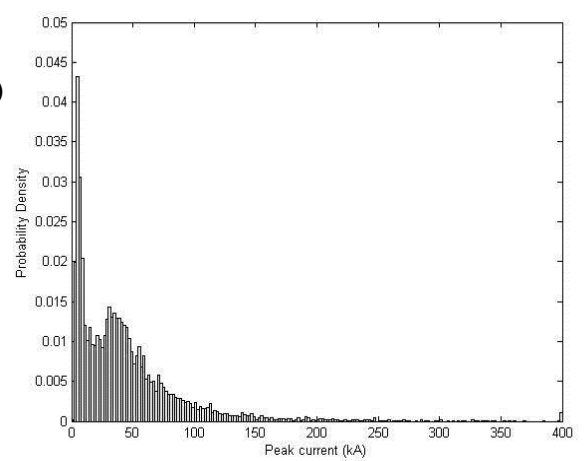

9

(c)

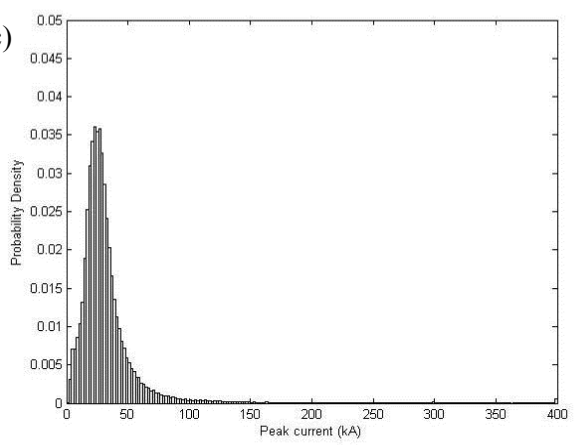

10

11 Fig. 1 Histogram of the peak current probability density of (a) the -CG lightning, (b) 
$1+$ CG lightning and $+\mathrm{IC}$ lightning identified from their peak currents less than $15 \mathrm{kA}$

2 and (c) the total CG lightning.

\section{Method}

The probability distribution of the LISSs is critical in estimating single-station acceptance. Being prior probability distribution, it can be deduced using samples of lightning location data. The likely probability distribution of these data would be that of either the Weibull, generalized extreme value (GEV), lognormal (LN), extreme value (EV) or gamma distribution. Hereinafter, we primarily introduce the GEV

9 distribution since it would provide the most accurate probability distribution fit of the

10 LISSs in the Beijing region.

21 with no restriction on $\mathrm{x}$.

22 The density distribution function is consequently

$23 f(x \mid \mu, \sigma, \xi)=\frac{1}{\sigma}\left[1+\xi\left(\frac{x-\mu}{\sigma}\right)^{(-1 / \xi)-1} \exp \left\{-\left[1+\xi\left(\frac{x-\mu}{\sigma}\right)\right]^{-1 / \xi}\right\}\right.$ for $1+\xi(x-\mu) / \sigma>0$, where $\mu \in R$ is the location parameter, $\sigma>0$ is the scale parameter and $\xi \in R$ is the shape parameter. Thus, for $\xi>0$, the CDF is only valid when $x>\mu-\sigma / \xi$; for $\xi<0$, the CDF is valid and has a short-tailed Gumbel distribution when $x<\mu+\sigma /(-\xi)$. For $\xi=0$, the CDF is formally undefined and can be replaced by the result obtained by taking the limit as $\xi \rightarrow 0$. Then,

$$
20 \quad F(x \mid \mu, \sigma, 0)=\exp \left[-\exp \left(-\frac{x-\mu}{\sigma}\right)\right]
$$


Nat. Hazards Earth Syst. Sci. Discuss., https://doi.org/10.5194/nhess-2017-307

Manuscript under review for journal Nat. Hazards Earth Syst. Sci.

Discussion started: 25 September 2017

(c) Author(s) 2017. CC BY 4.0 License.

1 When $\xi=0$, the density is positive over the entire real line and equal to the Weibull

2 distribution:

$3 f(x \mid \mu, \sigma, 0)=\frac{1}{\sigma} \exp \left[-\left(\frac{x-\mu}{\sigma}\right)\right] \exp \left\{-\exp \left[\left(-\frac{x-\mu}{\sigma}\right)\right]\right\}$

4

\subsection{Fundamentals of the GEV distribution applied for estimating single-station}

\section{acceptance}

Consider a normalized (for example, to a distance of $100 \mathrm{~km}$ ), undamped reference signal-strength distribution with the frequency function $f\left(s_{0}\right)$ and an expression for the signal strength dependence on the distance $s_{0}=D^{-1}(s)$. The simplest case for an undamped radiation field is the inverse power law $s=s_{0} r_{0} / r$, where $s_{0}=s r / r_{0}$. The acceptance can be calculated as a function of the distance and the lower and higher thresholds $s_{\min }$ and $s_{\max }$, respectively:

$$
\begin{aligned}
A(r) & =\int_{s D_{\min }}^{s D_{\max }} f\left(s_{0}\right) d s_{0}, s_{0}=D^{-1}(s), d s_{0}=D^{-1^{\prime}}(s) d s \\
& =\int_{s D_{\min }}^{s D_{\max }} f\left[D^{-1}(s)\right] D^{-1^{\prime}} d s=\left.F\left[D^{-1}(s)\right]\right|_{s_{\min }} ^{s_{\max }}
\end{aligned}
$$

For undamped propagation, we can obtain

$$
A(r)=F\left(s r / r_{0}\right) \mid{ }_{S_{\min }}^{s_{\max }}
$$

In the case of the GEV distribution, the acceptance is given by

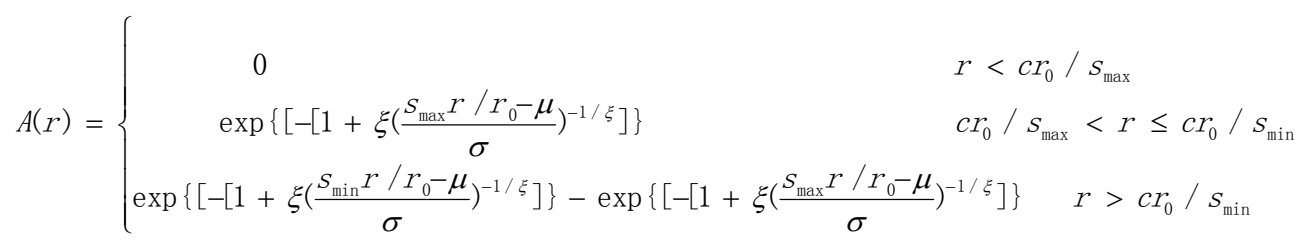

where $c$ is the signal bias and can be replaced by the minimum value of the signal samples.

$$
\text { The effective radius, } \rho \text {, describing the properties of a lightning counter or }
$$


Nat. Hazards Earth Syst. Sci. Discuss., https://doi.org/10.5194/nhess-2017-307

Manuscript under review for journal Nat. Hazards Earth Syst. Sci.

Discussion started: 25 September 2017

(c) Author(s) 2017. CC BY 4.0 License.

1 direction finder can be defined as

2

4

$$
=2 \int_{c r_{0} / s_{\min }}^{\infty} r \cdot \exp \left\{\left[-\left[1+\xi\left(\frac{S_{\max } r / r_{0}-\mu}{\sigma}\right)^{-1 / \xi}\right]\right\} d r-2 \int_{c r_{0} / s_{\max }}^{\infty} r \cdot \exp \left\{\left[-\left[1+\xi\left(\frac{S_{\min } r / r_{0}-\mu}{\sigma}\right)^{-1 / \xi}\right]\right\} d r\right.\right.
$$

8

$9 \quad$ By the substitution

10

11

12

$$
\int_{c r_{0} / s}^{\infty} r \cdot \exp \left\{\left[-\left[1+\xi\left(\frac{S r / r_{0}-\mu}{\sigma}\right)^{-1 / \xi}\right]\right\} d r\right.
$$

13

14

$$
\text { becomes }
$$

$$
\int_{0}^{\infty}\left(\frac{\sigma}{\xi \cdot S} z^{-\frac{1}{\xi}}+\frac{\sigma+\xi \cdot \mu \cdot r_{0}}{\xi \cdot S}\right) e^{-z} d z=\frac{1}{S}\left[\frac{\sigma}{\xi} \Gamma\left(\frac{-1}{\xi}\right)+\int_{0}^{\infty}\left(\frac{\sigma+\xi \cdot \mu \cdot r_{0}}{\xi}\right) e^{-z} d z\right]
$$

15 Thus, the substitution $A=\int_{0}^{\infty}\left(\frac{\sigma+\xi \cdot \mu \cdot r_{0}}{\xi}\right) e^{-z} d z$ does not include variable $s$ and is

16 integrable. The equation to calculate the effective radius can then be formulated as

$17 \rho^{2}=\left(1 / s_{\min }^{2}-1 / s_{\max }^{2}\right) \cdot\left[\frac{\sigma}{\xi} \Gamma\left(\frac{-1}{\xi}\right)+A\right]$

Because $S_{\min }$ is far less than $S_{\max }$, here, $S_{\min }$ can greatly influence the effective radius, while $S_{\max }$ has little influence on the effective radius.

\section{0 \\ 2.4 Damping modes}

(9) 
2 where Eq. (13) is calculated using the coefficient $k$ and the signal strength, $s$, has a

3 nonparametric unit (a.u.). The empirical value of the coefficient $k$ is $100 / 3$, equivalent

4 to a signal strength of 150 (a.u.) produced by a return stroke peak current of $45 \mathrm{kA}$

5 over a distance of $r_{0}=100 \mathrm{~km}$. The coefficient $p$ is also an empirical value, and here, it

6 is set to 1. Eq. (13) indicates a linear relationship between signal attenuation and the

7 signal propagating distance. Some applications prefer a nonlinear relationship; for

8 example, Cummins et al. (1998) attempted to determine an unbiased nonlinear

9 relationship to calculate the signal strength detected by the NLDN, in which $p$ was set

10 to 1.13 . However, our application found that the nonlinear and linear relationships

11 were similar (not given) for our results.

12 The lightning electromagnetic signal will be attenuated when propagated over

13 land and/or the ocean surface, and this attenuation is determined by the underlying

14 surface conductivity. Here, we introduce the parameter

$15 \gamma=r / \sigma$

16 where $r$ is the distance and $\sigma$ is the ground conductivity. The damping, $D$, is

17 expressed as

$18 D(\gamma)=1.652-0.0491 \ln \left(\gamma / \gamma_{0}\right)$

19 in which

$20 \gamma>10^{6} m^{2} \Omega, \gamma_{0}=1 m^{2} \Omega$

21 The damping factor $D$ is multiplied by the $1 / r$ propagation law for the radiation fields

22 in order to calculate the signal-strength

$23 s(r)=D(r / \sigma)_{S_{0}} r_{0} / r$

24 Therefore, in the damping modes, the single-station acceptance can be calculated as

$25 A(r)= \begin{cases}0 & r<c r_{0} / s_{\max } \\ \exp \left\{\left[-\left[1+\xi\left(\frac{D(r / \sigma) s_{\max } r / r_{0}-\mu}{\sigma}\right)^{-1 / \xi}\right]\right\}\right. & c r_{0} / s_{\max }<r \leq c r_{0} / s_{\min } \\ \exp \left\{\left[-\left[1+\xi\left(\frac{D(r / \sigma) s_{\min } r / r_{0}-\mu}{\sigma}\right)^{-1 / \xi}\right]\right\}-\exp \left\{\left[-\left[1+\xi\left(\frac{D(r / \sigma) s_{\max } r / r_{0}-\mu}{\sigma}\right)^{-1 / \xi}\right]\right\}\right.\right. & r>c r_{0} / s_{\min }\end{cases}$ 


\subsection{Iterative algorithm for DE estimation}

After the acceptance of each detector in a lightning location network is confirmed, the DE of the network can be estimated using an iterative algorithm. The detector location modes are determined by lightning locating methods, e.g., MDF and TOA.

The MDF method requires at least two detectors synchronously detecting one lightning return stroke (Schütte et al. 1987), while the TOA method requires four detectors since the signals received by two additional TOA detectors are needed to eliminate the falsely duplicated locations detected by the other two detectors. The IMPACT detector networks locate a lightning stroke using at least two detectors. Thus, an iterative algorithm is needed in the deduction of DEs, especially with respect to lightning locations that require three or more detectors in LLS network coverage.

The algorithm is expressed as follows.

1) This iterative algorithm is designed for $\mathrm{DE}$ estimation in two-detector networks:

where $F(2,2)$ is the $\mathrm{DE}$ of the two-detector location mode in a two-detector network and $A\left(r_{1}\right)$ and $A\left(r_{2}\right)$ are acceptances of the detectors $r_{l}$ and $r_{2}$, respectively.

2) In three-detector networks

where $F(3,2)$ is the $\mathrm{DE}$ of the two-detector location mode in a three-detector network, $A\left(r_{2}\right)$ is the acceptance of detector $r_{3}$, and $R\left(r_{1}\right), R\left(r_{2}\right)$ and $R\left(r_{3}\right)$ are the non-acceptances of detectors $r_{1}, r_{2}$ and $r_{3}$, respectively.

3) In an $n$-detector network $(n>3)$,

28 In Eq. (20), 
$2 \quad 3$ Test and case study

$3 \quad 3.1$ Identifying the probability distribution and estimating parameters

The maximum likelihood method allows the determination of the most effective estimators, and the sample data histogram is helpful in identifying the probability distribution (Bishop 2006; Kotowski and Zmierczak 2013). The sample data of 240804 lightning CG strokes detected by the ADTD network from 2007 to 2016 were used to determine the probability distribution of the LISSs in Beijing and the parameters of the probability density function (PDF). To obtain the fittest probability distribution, which can be used to calculate the probability above a certain signal strength threshold, we estimated the parameters of the Weibull, the lognormal, the extreme value, the gamma, and the generalized extreme value (GEV) distributions

13 from the sample data. In a comparison of the fitness of these probability distribution 14 curves and the sample data histogram, the GEV curve is identified as the best fitted 15 (Fig. 2). Therefore, the GEV probability distribution is regarded as the distribution with the best fit and is used in deducing single-station acceptance. The probability distribution of the LISSs is empirically established in certain regions, and it should be properly predefined before applying the results to deduce acceptance.

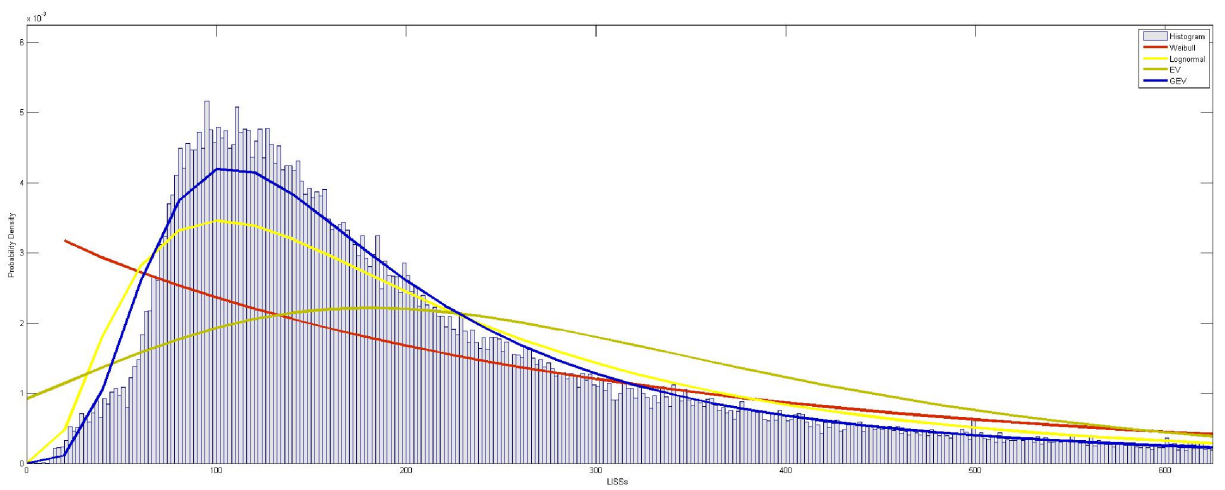

Fig. 2 Fitted probability density function curves for (a) the Weibull (red line), (b) the log normal (light yellow line), (c) the extreme value (deep yellow line) and (d) the GEV (blue line) distributions of the LISSs with the histogram of the sample data. 


\subsection{DE estimates}

The ADTD system observes the lightning parameters of the CG lightning strokes, e.g., the peak current, current intense slope, and latitude and longitude of lightning stroke position. However, the CG lightning strokes were not grouped into lightning flashes by origin. We estimated the stroke DEs rather than the flash DEs.

Usually, if the first return stroke or one of its subsequent return strokes in a multiple lightning flash is located, that flash can be counted as detected (Naccarato and Pinto 2009). Therefore, the stroke DEs would be lower than the flash DEs. Naccarato and Pinto (2009) confirmed that the ratio of the stroke DEs to the flash DEs

11 in the Paraíba Valley, Brazil, should be 1.6, implying a larger magnitude of stroke 12 DE.

We deduced the parameters of the GEV distribution of the LISSs using the sampled lightning electromagnetic signal data (Fig. 1): $\sigma=97.449, \xi=0.5083$, $\mu=143.2815$. Next, the single-station signal acceptance was deduced using Eq. (17).

When dealing with the signal attenuation induced by underlying land surface conductivity, we used the soil-type distribution in the Beijing region to estimate the damping coefficient in the grids. The Beijing Bureau of Geology and Mineral Resources (BBGMR) provided the soil-type distribution maps, which had been digitized as GIS polygons of the soil type (not displayed here). These polygons can be overlapped with the propagating paths of electromagnetic lightning signals to obtain the path segments over different soil-type polygons, which exhibit different conductivities and attenuation signals. The predefined soil-type conductivities are listed in Table 1. Additionally, we generalized the LCLU as regions of mountains and plains where we lacked the detailed soil-type distribution. Accounting for the most extensively distributed bare rocks, gravels, and sands in the mountainous areas, which exhibit lower conductivity, the mountainous conductivity magnitude is simplified to be $1500 \Omega . \mathrm{m}$. On the other hand, the conductivity in the plains, which are mostly composed of loamy soil, is $60 \Omega . \mathrm{m}$. Therefore, using Eq. (14) to Eq. (17), we can 
Nat. Hazards Earth Syst. Sci. Discuss., https://doi.org/10.5194/nhess-2017-307

Manuscript under review for journal Nat. Hazards Earth Syst. Sci.

Discussion started: 25 September 2017

(C) Author(s) 2017. CC BY 4.0 License.

(c) (1)
Natural Hazards and Earth System

Sciences

Discussions

$-14-$

1 estimate the signal strength influenced by the damping effects induced by the land

2 surface conductivity.

3

4

Table 1 Lists of underlying soils conductivities.

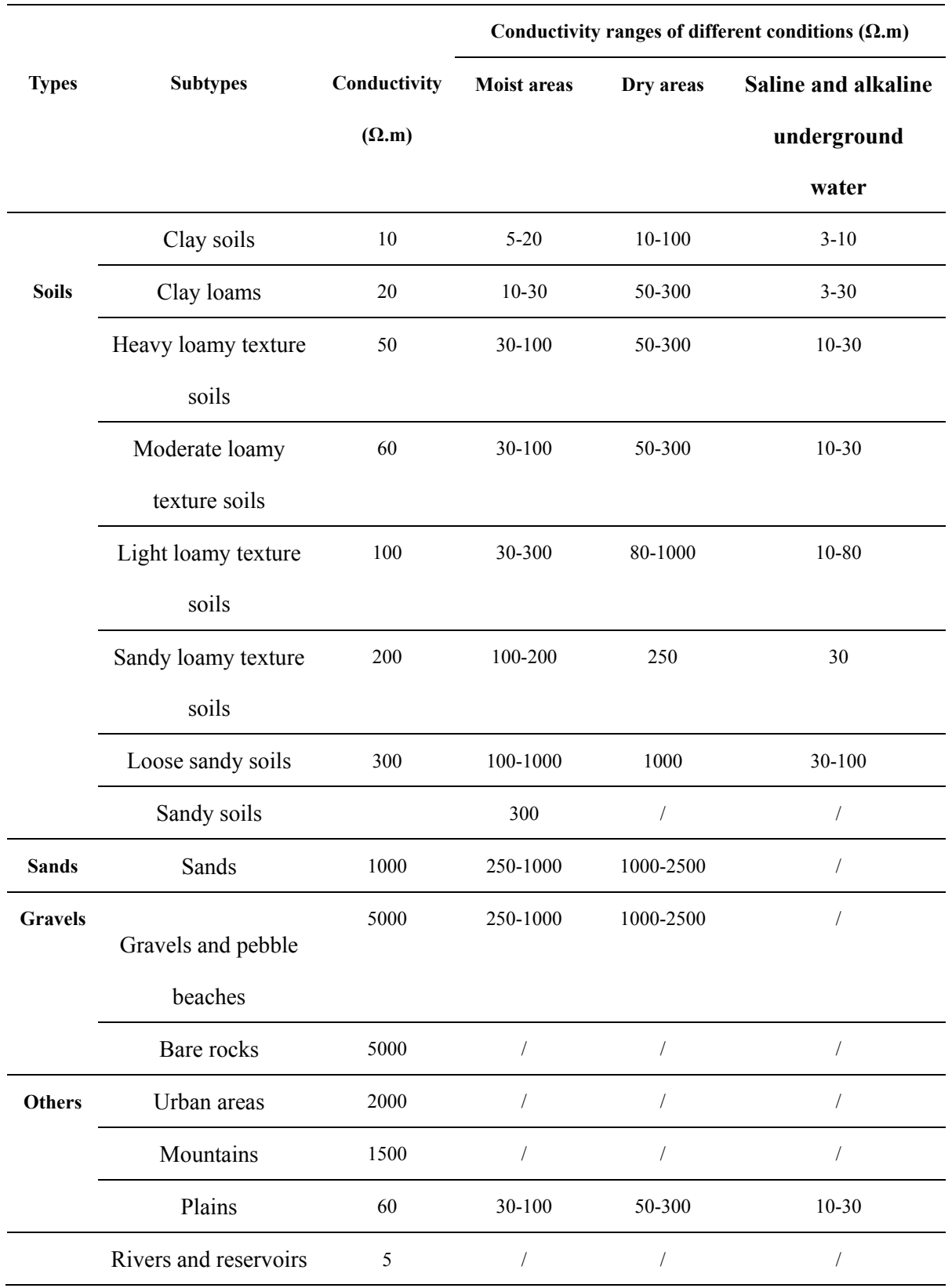


Nat. Hazards Earth Syst. Sci. Discuss., https://doi.org/10.5194/nhess-2017-307

Manuscript under review for journal Nat. Hazards Earth Syst. Sci.

Discussion started: 25 September 2017

(c) Author(s) 2017. CC BY 4.0 License.
Natural Hazards and Earth System

Sciences

Discussions

(c) (1)

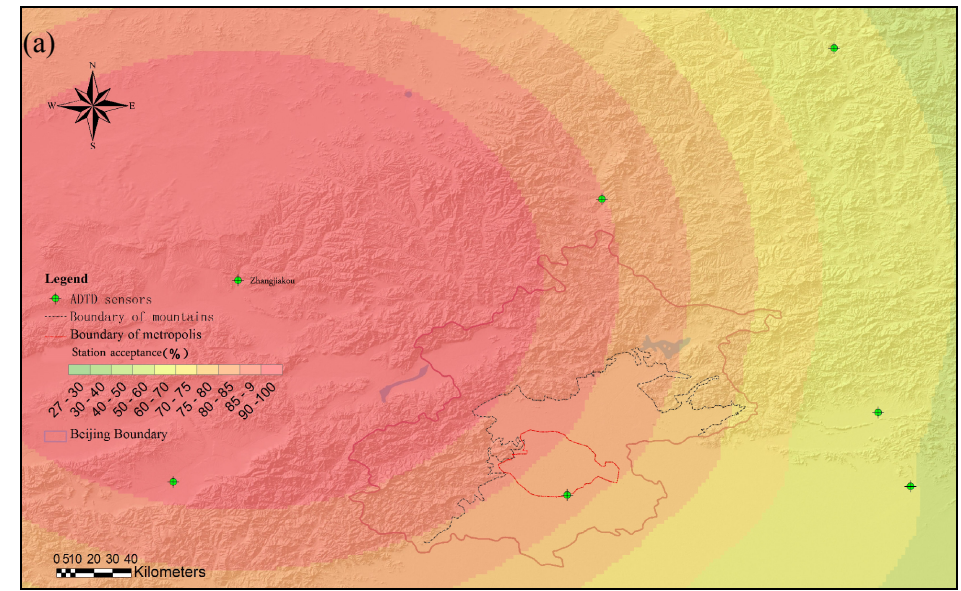

2
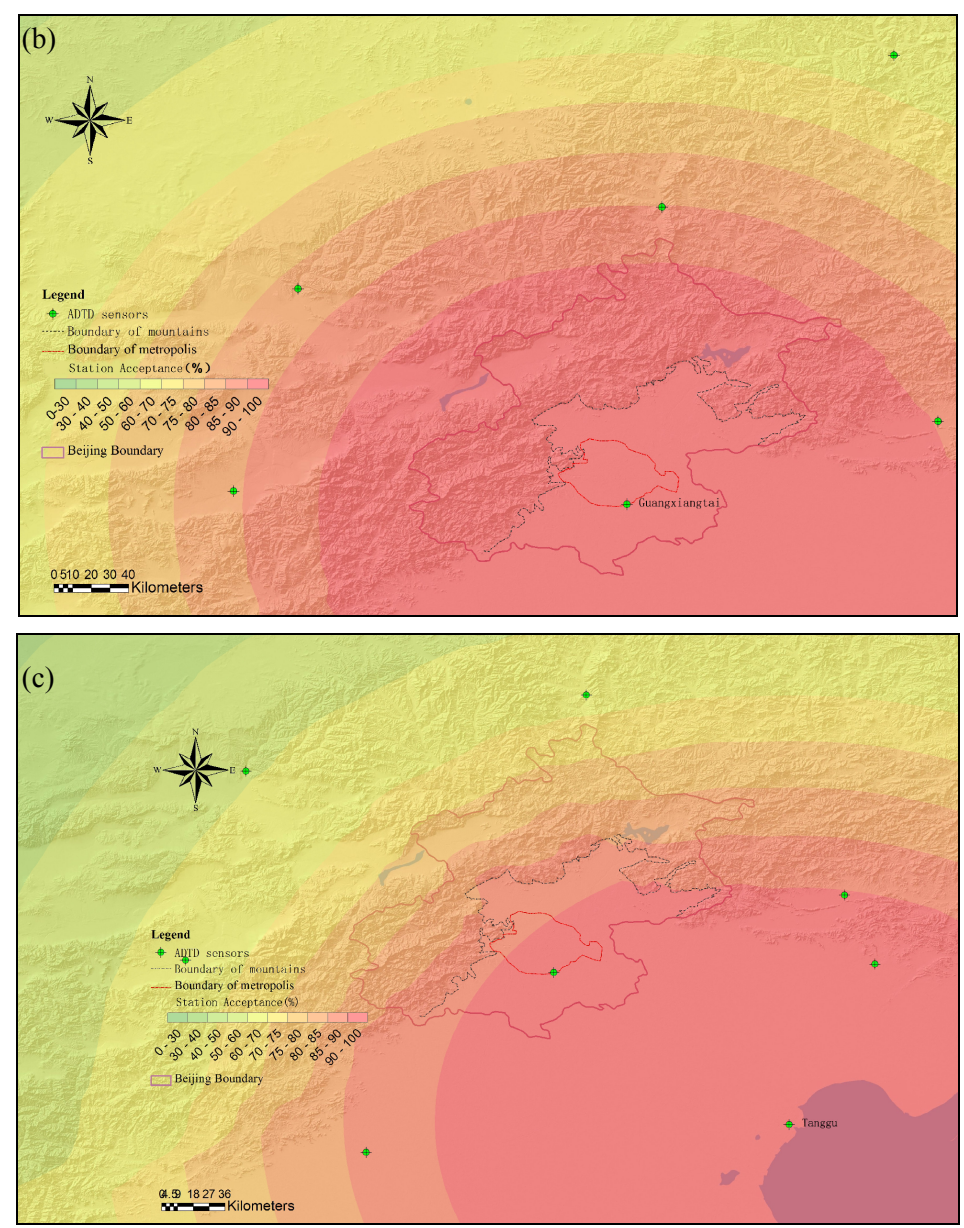

Fig. 3 Distributions of the single-station acceptances of the detectors in (a)

5 Zhangjiakou, (b) Guangxiangtai, and (c) Tanggu. 
Using Eq. (17), we calculated the single-station acceptances $A(r)$ of approximately 11 ADTD detectors around Beijing. The distribution maps indicate that the acceptances in areas closer to the detectors are clearly higher and that the acceptances rapidly decrease toward the mountainous areas (see Fig. 3).

Subsequently, we deduced the ADTD network DE (Fig. 4) using the iterative algorithm based on single-station acceptances. According to the recordings of the location modes in the ADTD data detected from 2007 to 2016, these CG lightning strokes were located after their signal sources were received synchronously by 2 to 7

9 detectors (see Table 2 for the proportions of each location mode). Note that only 11

10 ADTD detectors in the Beijing region had been selected to estimate the DE for the case study, and thus, the results are only valid in this region. The results indicate that the DEs in the Beijing metropolitan area are relatively higher, almost all are above $60 \%$, and the maximum is $78 \%$ (see Fig. 4 ).

It is reasonable to expect that the CG lightning stroke DEs can reach $60 \%$ or higher. Based on a video observation, Idone (1998) found that from 1994 to 1996, the in-site NLDN stroke DEs were most likely between $47 \%$ and $67 \%$, while the flash DEs reached between $72 \%$ and $86 \%$. Regarding the Vaisala reports, Orville et al. (2001) suggested that the flash DEs of the North American Lightning Detection Network (NALDN) in the contiguous U.S. and southern Canada should surpass 90\%, while the overall stroke DEs would be between $60 \%$ and $80 \%$.

According to the DE estimates of the ADTD network (Fig. 4), in contrast to the low DEs in the mountainous northern and western regions, the southern plains exhibit a higher DE. Relatively exceptionally low DEs can be found in parts of the Beijing district. These areas are closer to the detector at Guangxiangtai station but far away from the other closest detectors (e.g., Zhangjiakou), meaning that the signal would propagate a long distance over mountains before being received by these detectors; as a consequence, the signal would be attenuated.

We estimated the DEs of the ADTD network by both accounting and not accounting for the damping effects induced by the underlying conductivity. In the comparison of the DEs deduced in the damping and nondamping modes, the DE 
Nat. Hazards Earth Syst. Sci. Discuss., https://doi.org/10.5194/nhess-2017-307

Manuscript under review for journal Nat. Hazards Earth Syst. Sci.

Discussion started: 25 September 2017

(c) Author(s) 2017. CC BY 4.0 License.
Natural Hazards

and Earth System

Sciences

Discussions

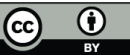

$-17-$

1 deduced in the nondamping mode is clearly higher than that in the damping mode, and

2 this discrepancy is exaggerated in mountainous areas (see Fig. 4), where the lower

3 conductivity leads to a greater attenuation of lightning electromagnetic signals.

4

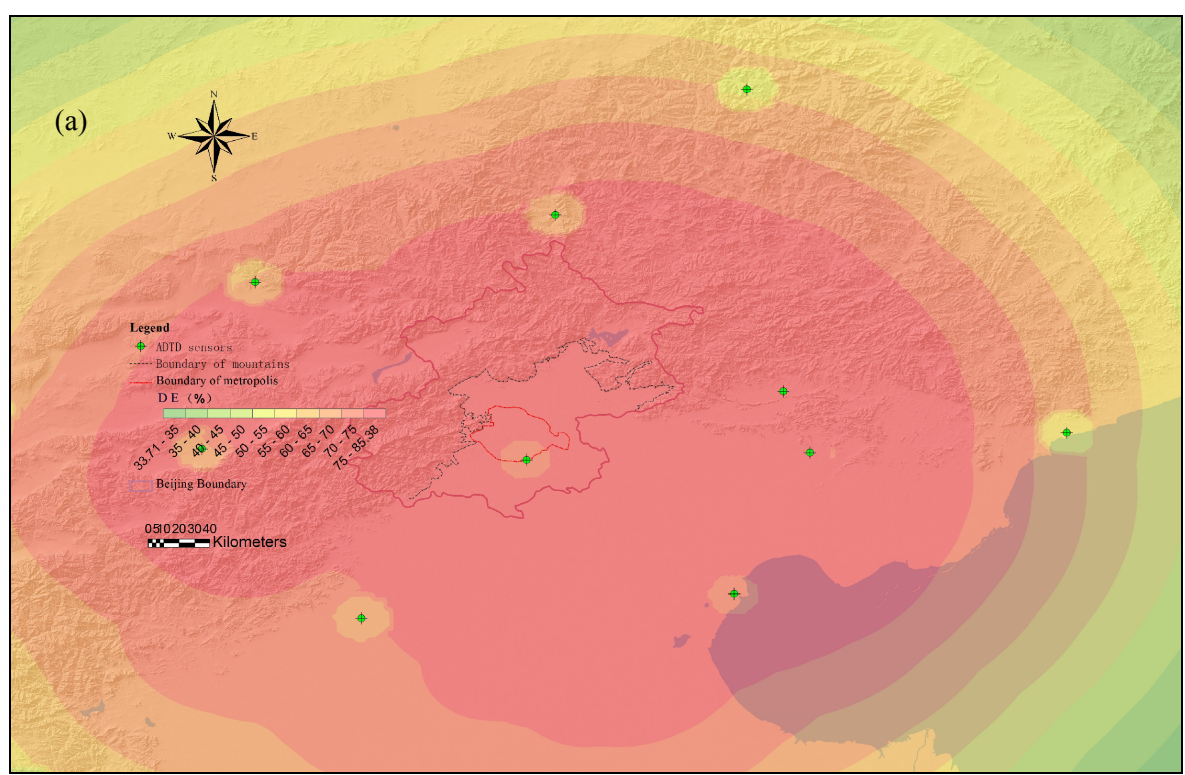

(b)
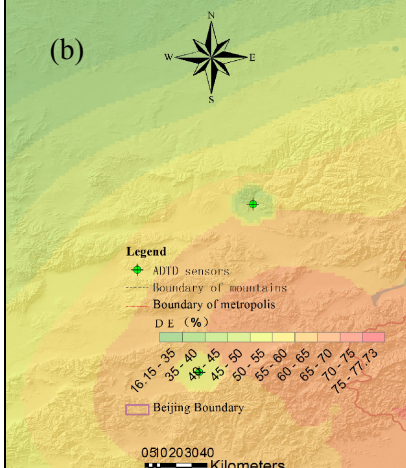

0510203040 Kilometers

6 Fig. 4 Deduced CG lightning stroke DE of the ADTD network around Beijing (a) not

7 accounting for the land surface conductivity damping and (b) accounting for the land

8 surface conductivity damping. 
2 Table 2 Proportions of the ADTD location modes recorded in the lightning

3 location data

\begin{tabular}{lllllll}
\hline $\begin{array}{l}\text { Location } \\
\text { modes }\end{array}$ & $\begin{array}{l}\text { 2-sensor } \\
\text { location }\end{array}$ & $\begin{array}{l}\text { 3-sensor } \\
\text { location }\end{array}$ & $\begin{array}{l}\text { 4-sensor } \\
\text { location }\end{array}$ & $\begin{array}{l}\text { 5-sensor } \\
\text { location }\end{array}$ & $\begin{array}{l}\text { 6-sensor } \\
\text { location }\end{array}$ & $\begin{array}{l}\text { 7-sensor } \\
\text { location }\end{array}$ \\
\hline Proportion & $34.25 \%$ & $2.45 \%$ & $4.3 \%$ & $19.18 \%$ & $37.6 \%$ & $2.22 \%$
\end{tabular}

\subsection{CG lightning stroke density corrected for the deduced DEs}

The CG lightning stroke densities (stroke/yr.km²) were derived from the ADTD lightning location data. The derivation was performed on the $1 \times 1 \mathrm{~km}$ resolution grid, which is $182 \mathrm{~km} \mathrm{~N}-\mathrm{S}$ and $181 \mathrm{~km} \mathrm{E-W}$ and covers the entire Beijing district. The GIS "contain" operator is applied for counting the lightning CG stroke numbers in the grid cells. Then, the CG lightning stroke density $N_{\text {grid }}$ can be corrected for the grid DEs $F_{\text {grid }}$, and the correction formula is

$$
N_{\text {grid }}^{\prime}=N_{\text {grid }} / F_{\text {grid }}
$$

where $N_{\text {grid }}^{\prime}($ stroke/yr.km²) is the corrected CG lightning stroke density.

The derivation indicated that the CG lightning stroke densities in the mountainous northern and western regions are clearly lower than that of the other areas (see Fig. 5). This characteristic is similar to that of the DE estimates. In the mountainous regions, the elevated terrains and low conductivity of the underlying surface have a strong influence on signal attenuation (Cummins et al. 2006), which in turn reduces the DE and location accuracy of the ADTD network. However, this result does not convincingly prove that the low CG lightning stroke density in mountainous areas is determined by the low DEs.

After the CG lightning stroke density was corrected for the DE, the densities in the mountainous northern and western regions increased slightly but remained lower than those of the other areas (see Fig. 5). Higher densities are still present in the mountainous areas of eastern and southwestern Beijing and in the plains.

A high CG stroke density zone appears in the urban area and its downwind areas (see A in Fig. 5b), in agreement with the hypothesis that an increased urban land-surface roughness could intensify thunderstorm activities (Shepherd et al. 2002; 
1 Rose 2008; Stallins et al. 2006; Stallins and Rose 2008; Hu et al. 2014, 2015; Sun et 2 al. 2007).

Additionally, the aerosols serve as cloud condensation nuclei (CCN) and have a 4 substantial effect on cloud properties and the initiation of precipitation (Steiger and 5 Orville 2002; Naccarato et al. 2003). The higher CG lighting strikes predicted in the 6 plains agree with the hypothesis of the urban aerosol effects on lightning enhancement 7 since the plains usually have more polluted air than the mountainous areas (Tie et al. 8 2015). Moreover, an urban heat island (UHI) can trigger an urban-to-rural thermal 9 circulation and force a convection, which produces enhanced thunderstorms in the 10 metropolitan regions (Jiang et al. 2006; Sun et al. 2007).

11 Therefore, it is justifiable that the corrected CG lightning stroke densities would 12 be higher in the plains, almost twice (mostly above 2.0 stroke/ $\mathrm{yr}^{\mathrm{k}} \mathrm{km}^{2}$ ) the density in 13 the northern and northwestern areas. Therefore, the urbanization in the plains plays an 14 important role in inducing thunderstorm enhancement that results in a higher CG lightning stroke density without accounting for the higher DEs in the plains.

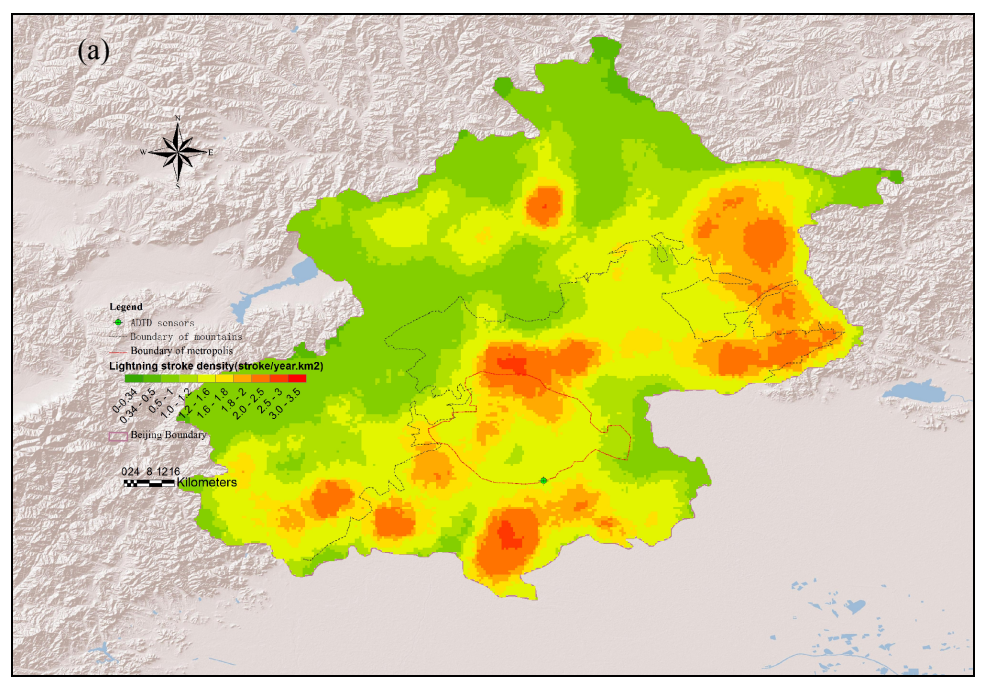




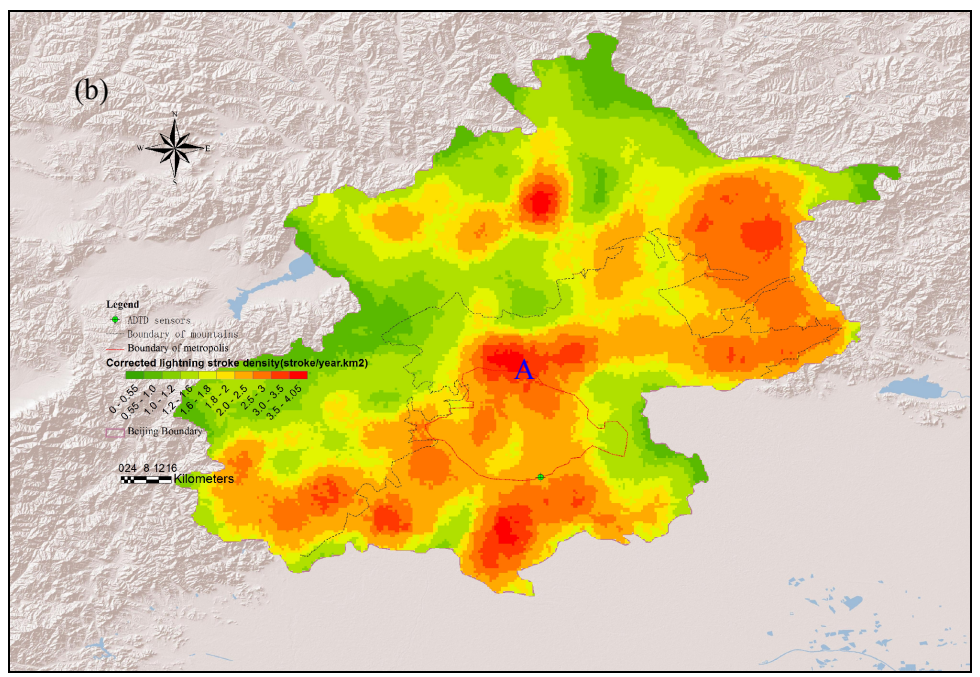

Fig. 5 Distributions of the ADTD-derived (a) non-corrected CG lightning stroke density and (b) CG lighting stroke density corrected for the DE.

\section{Conclusions}

The DE is determined by the LLS performance and sensitivity of the detectors, detector network geometry, and damping factors induced by the underlying land surface conductivity. For this reason, DEs vary due to the underlying land surface and become very low in some regions. This situation leads to a discrepancy between the derived and actual lightning flash/stroke density. This characteristic discounts the applicability of the LLS data. Therefore, we developed an algorithm to estimate DES which can be used for lightning flash/stroke density correction. The method and its implementation in the CG lightning stroke density correction can be summarized as follows:

(1) It is critical to identify a suitable probability distribution of the LISSs, which is determined for specific regions. In comparison with the lognormal, the Weibull, the extreme value $(\mathrm{EV})$ and the GEV probability distributions, we found that the GEV distribution provides the best fit to the histogram of the sample data.

(2) Based on formula derivations, it was verified that the GEV probability distribution fit would be suitable in estimating the DEs and that the single-station 
effective radius and acceptance would be determined by the minimum detectable signal strength $\left(S_{\min }\right)$.

(3) To address the damping effects induced by the inconsistent underlying land surface conductivity, we estimated the single-station acceptance in the damping modes with respect to the GEV probability distribution of the LISSs.

(4) The DEs of the ADTD network were deduced based on an iterative algorithm using single-station acceptances. According to the LLS location data in Beijing, the number of detectors required to locate the CG lightning strokes was generally between 2 and 7. Thus, the complexity of the iterative algorithm was low, and a convergent solution was found.

(5) The damping effects induced by underlying land surface conductivity were considered in the DE estimates. Thus, the estimate results exhibit that the DEs in the mountains are clearly lower than that in the plains. This finding may be due to the lightning electromagnetic signals propagating over mountainous areas with lowconductivity underlying land surfaces, which considerably attenuates the signal.

(6) The CG lightning stroke density corrections for the DEs reflected that although the densities in the mountainous northern and western areas increase to some degree, they are still lower than those in the highly urbanized plains. This phenomenon can be related to the increased urban land-surface roughness, the aerosols and the UHI effects on the thermodynamic activity in urban areas.

The DE estimates can be effectively used for correcting the CG lightning 22 flash/stroke density derived from the LLS data. However, we suggest that the location error correction and uncertainty reduction in the lightning detection should be continued by adding new detectors and/or network upgrading and optimizing the network sensor deployment. This approach would vitally enhance the LLS performance and sensor sensitivity and achieve the goal of DE improvement. 
This study has been supported by the Climate Change Research Projects of China of Urban Meteorology, CMA (Project: IUMKY201716).

\section{Reference}

6 Antonescu, B., and S. Burcea, 2010. A Cloud-to-Ground Lightning Climatology for

7 Romania. Mon. Wea. Rev., 138, 579-591. doi:

8 http://dx.doi.org/10.1175/2009MWR2975.1.

9 Bourscheidt, V., Cummins, K. L., Pinto, Jr., O., and K. P. Naccarato, 2012. Methods to

10 Overcome Lightning Location System Performance Limitations on Spatial and 11 Temporal Analysis: Brazilian Case., J. Atmos. Oceanic. Technol., 29, 1304-1311. Bishop, C. M., 2006: Pattern recognition and machine learning. Springer Science+Business Media, LLC, Singapore. Changnon, S.A., 1993. Relationships between Thunderstorms and Cloud-to-Ground Lightning in the United States. J. Appl. Meteor., 32, 88-105. Cummins, K.L., Bardo, E.A., Hiscox,W.L., Pyle, R.B., Pifer, A.E.,1995. NLDN'95: a combined TOA/MDF technology upgrade of the U.S. National Lightning Detection Network. Proceedings of the International Aerospace \& Ground Conference on Lightning and Static Electricity. National Interagency Coordination Group, Williamsburg.

Cummins, K. L., J. A. Cramer, W. A. Brooks, and E. P. Krider, 2005: On the effect of land-sea and other earth surface discontinuities on LLS-inferred lightning parameters. Proc. Eighth Int. Symp. On Lightning Protection, Sao Paulo, Brazil, Institute of Electrotechnics and Energy of the University of Sa o Paulo, 106-111. Cummins, K.L., Murphy, J.M., Bardo, E.A., Hiscox, W.L., Pyle, B.R., Pifer, A.E., 1998. A Combined TOA/MDF Technology Upgrade of the U.S. National Lightning Detection Network. J. Geophys. Res., 103(D8), 9035-9044. 
history, techniques, and data uses, with an in depth look at the US NLDN, IEEE T.

2 Electromagn. C., 51, 499-518.

3 Drüe, C., Hauf, T., Finke, U., Keyn, S., and Kreyer, O., 2007. Comparison of a SAFIR

4 lightning detection network in northern Germany to the operational BLIDS network. J.

5 Geophy. Res., 112(D18114), doi:10.1029/2006JD007680.

6 Etherington, T. R., and Perry, G. L.W., 2017. Spatially adaptive probabilistic

7 computation of a sub-kilometre resolution lightning climatology for New Zealand.

8 Computers \& Geosciences, 98: 38-45, 2017.

9 Fierro, A. O., E. R., Mansell, C. L., Ziegler, and D. R., Macgorman, 2011:Application

10 of a Lightning Data Assimilation Technique in the WRF-ARW Model at

11 Cloud-Resolving Scales for the Tornado Outbreak of 24 May 2011. Mon. wea.

12 Rev., 140, 2609-2627.

13 Fierro, A. O., and J. M. Reisner, 2011: High-resolution simulation of the 14 electrification and lightning of Hurricane Rita during the period of rapid 15 intensification. J. Atmos. Sci., 68, 477-494. Honma, N., F. Suzuki, Y. Miyake, M. Ishii, and S. Hidayat, 1998: Propagation effect on the field waveforms on relation to TAO technique in lightning location. J. Geophys. Res., 103, 14 141- 14146.

Hu, H., Wang, J., Pan, J., 2014. The characteristics of lightning risk and zoning in Beijing simulated by a risk assessment model. Nat. Hazards Earth Syst. Sci ～， 14, 21 1985-2014. doi:10.5194/nhess-14-1985-2014.

$22 \mathrm{Hu}, \mathrm{H} ., 2015:$ Spatiotemporal Characteristics of Rainstorm-Induced Hazards Modified

23 by Urbanization in Beijing. J. Appl. Meteorol. Climatol., 54(7):1496-1509.

24 Idone, V.P., Davis, D.A., Moore, P.K., Wang, Y., Henderson, R.W., Ries, M., Jamason, P.F., 1998. Performance evaluation of the U.S. National Lightning

Res., 103, 9045-9056, doi:10.1029/ 98JD00154. 
1 Kotowski, A., and Kazmierczak, B., 2013. Probabilistic Models of Maximum

Precipitation for Designing Sewerage, J. Hydrometeo., 14, 1958-1965.

Ma, Q. M., 2015: Technology and fundamentals of lightning monitor. Science press, Beijing. (in Chinese)

Mäkelä, A., Tuomi, T.J., Haapalainen, J., 2010. A decade of high - latitude lightning location: Effects of the evolving location network in Finland. J. Geophy. Res., 115(D21124), doi:10.1029/2009JD012183.

Naccarato, K. P., Pinto, O., and Pinto, I., 2003. Evidence of thermal and aerosol effects on the cloud-to-ground lightning density and polarity over large urban areas of Southeastern Brazil. Geophys. Res. Let. 30, pp. 1674-1677.

Naccarato, K.P., Pinto, Jr., O., 2009. Improvements in the detection efficiency model for the Brazilian lightning detection network (BrasilDAT). Atmos. Res., 91, 546-563. Orville, R. E., G. R. Huffines, W. Burrows, R. Holle, and K. Cummins. The North American LightningDetection Network (NLDN) First results: 1998-2000. Mon. Wea. Rev., 2002, 130: 2098-2108. doi: 10. 1175/1520-0493(2002)130, 2098: TNALDN.2.0.CO; 2.

Orville, R. E., Huffines, G. R., Burrows, G. R., Cummins, K. L., The North American Lightning Detection Network (NALDN)_Analysis of Flash Data: 2001-09. Mon. wea. rev., 2001, 139:1305-1322.

Rakov, V. A., and Uman, M. A.: Some properties of negative cloud-to-ground lightning flashes versus strike order. J. Geophys. Res., 95(D5): 5447- 5453, 1990.

Rose, L.S., Stallins, J.A., Bentley, M.L., 2008. Concurrent cloud-to-ground lightning and precipitation enhancement in the Atlanta, Georgia (United States), urban region, Earth Interact., 12, 1-30, doi:10.1175/2008EI265.1.

Rudlosky, S., and Fuelberg, H.E., 2010. Pre- and Postupgrade Distributions of NLDN

Reported Cloud-to-Ground Lightning Characteristics in the Contiguous United States.

Mon. Wea. Rev., 138, 3623-3633.

8 Schulz, W., Cummins, K.L., Diendorfer, G., Dorninger, M., 2005. Cloud-to-ground

9 lightning in Austria: A 10-year study using data from a lightning location system. J.

0 Geophy. Res., 110, D09101. doi:10.1029/2004JD005332, 2005. 
Schütte, T., Salka, O., Israelsson, S., 1987. The use of the Weibull distribution for thunderstorm parameters. J. Climate and Applied Meteorology, 26, 457-463. system acceptance using a refined damping model, J. Atmos. Oceanic. Technol., 5, $5 \quad 375-380$.

6 Shepherd, J.M., Pierce, H., Negri, A.J., 2002. Rainfall modification by major urban

7 areas: observations from spaceborne rain radar on the TRMM satellite, J. Appl.

8 Meteorol., 41, 689-701, 2002.

9 Sun, J.S., and Shu, W.J., 2007:The Effect of Urban Heat Island on Winter and

10 Summer Precipitation in Beijing Region. Chinese Journal of Atmospheric Sciences (in

11 Chinese), 2007, 31(2):311-320.

12 Stallins, J.A., Bentley, M.L., Rose, L.S., 2006. Cloud-to-ground flash patterns for 13 Atlanta, Georgia (USA) from 1992 to 2003. Climate Res., 30, 99-112.

14 Stallins, J.A., and L.S. Rose, 2008. Urban lightning: current research, methods, and

15 the geographical perspective, Geography Compass, 2, 620-639,

16 doi:10.1111/j.1749-8198.2008.00110.x, 2008.

17 Steiger, S.M., Orville, R.E., Huffines, G., 2002. Cloud-to-ground lightning

18 characteristics over Houston, Texas: 1989-2000. J. Geophys. Res., 107, 4117, 19 doi:10.1029/2001JD001142.

20 Taszarek, M., and B. Czernecki. A Cloud-to-Ground Lightning Climatology for 21 Poland. Mon. Wea. Rev., 2015, 143:4285-4304.

22 Tie, X., Q. Zhang, H. He, J. Cao, S. Han, Y. Gao, X. Li, X. C. Jia, 2015. A budget analysis of the formation of haze in Beijing. Atmospheric Environment, 100: 25-36. Williams, E.R., 2005. Lightning and climate: a review. Atmos. Res., 76, 272-287. Yao, W., Zhang, Y., Meng, Q., Wang, F., Lu, W., 2012. A Comparison of the 